\title{
Oxydation de la matière organique dans un système hydrologique karstique alimenté par des pertes fluviales (Loiret, France)oxidation of organic matter in a karstic hydrologic unit supplied through stream sinks (Loiret, France)
}

\author{
P. Albéric ${ }^{\mathrm{a}}$ M. Lepiller ${ }^{\mathrm{b}}$
}

- $\quad{ }^{\text {a }}$ Laboratoire de Géochimie organique, CNRS UMR 6531 et FR 09, Université d'Orléans, 45100 Orléans, France

- $\quad{ }^{\mathrm{b}}$ Laboratoire d'Hydrogéologie, Université d'Orléans, 45100 Orléans, France

\section{Résumé}

L'impact de l'oxydation de la matière organique fluviatile sur la dissolution du calcaire a été mesuré pendant le transit des eaux du fleuve Loire à travers le système karstique du val d'Orléans. Les caractéristiques physico-chimiques des eaux à l'entrée et à la sortie du système ont été étudiées en période estivale (mai à octobre) en particulier pendant des épisodes de crue. La variation des teneurs en chlorure équivaut à un traçage naturel et global des pertes du fleuve qui confirme les temps de transit courts (environ 3 jours) déterminés antérieurement à l'aide de traceurs artificiels. En fonction du régime du fleuve, les charges organiques dissoute et particulaire varient respectivement de 3 à 7 et de 2 à $13 \mathrm{mg} \mathrm{C} / \mathrm{l}$. Pendant le transit souterrain, matière organique et oxygène dissous diminuent, tandis que calcium et $\mathrm{C}$ minéral augmentent. Le bilan de l'oxydation de la matière organique montre qu'en période estivale, environ $65 \mu \mathrm{g} \mathrm{C}_{\mathrm{org}} / \mathrm{l} \cdot \mathrm{h}$ sont oxydés, avec peu de variation en fonction du régime du fleuve ou de sa charge organique. Le calcul montre également que la dissolution du calcaire dépend strictement de l'oxydation de la matière organique fluviatile introduite, tout en variant (7$29 \mathrm{mg} \mathrm{CaCO}_{3} / \mathrm{l}$ ) en fonction des caractéristiques acido-basiques des eaux de Loire entrant dans le système.

\section{Abstract}

The aim of this paper is to appraise the ability of the oxidation of riverine organic matter in the control of limestone dissolution, in a karst network. Biogeochemical processes during infiltration of river water into an alluvial aquifer have already been described for an average flow velocity of 4-5 m d ${ }^{-1}$ (Jacobs, L. A., von Gunten, H. R., Keil, R. and Kuslys, M. (1988) Geochemical changes along a river-groundwater infiltration flow path: Glattfelden, Switzerland. Geochim. Cosmochim. Acta52, 2693-2706; Von Gunten, H. R., Karametaxas, G., Krähenbühl, U., Kuslys, M., Giovanoli R., Hoehn E. and Keil R. (1991) Seasonal biogeochemical cycles in riverborne groundwater. Geochim. Cosmochim. Acta55, 3597-3609; Bourg, A. C. M. and Bertin, C. (1993) Quantitative appraisal of biogeochemical processes during the infiltration of river water into an alluvial aquifer. Environ. Sci. Technol. 27, 661666). Karstic drainage networks, such as in the River Loire-Val d'Orléans hydrologic system (Fig. 1), make possible flow velocities up to $200 \mathrm{~m} \mathrm{~h}^{-1}$ and provide convenient access to different water samples several tens of $\mathrm{km}$ apart, at both extremities of the hydrologic unit (Chéry, J.-L. (1983) Etude hydrochimique d'un aquifère karstique alimenté par perte de cours d'eau (la Loire): Le système des calcaires de Beauce sous le val d'Orléans. Thèse, Université d'Orléans; Livrozet, E. (1984) Influence des apports de la Loire sur la qualité bactériologique et chimique de l'aquifère karstique du val d'Orléans. Thèse, Université d'Orléans). Recharge of the karstic aquifer occurs principally from influent waters from stream sinks, either through 
coarse alluvial deposits or directly from outcrops of the regional limestone bedrock (Calcaires de Beauce). Recharge by seepage waters from the local catchment basin is small (Zunino, C., Bonnet, M. and Lelong, F. (1980) Le Val d'Orléans: un exemple d'aquifère à alimentation latérale. C. R. somm. Soc. Géol. Fr. 5, 195-199; Gonzalez R. (1992) Etude de l'organisation et évaluation des échanges entre la Loire moyenne et l'aquifère des calcaires de Beauce. Thèse, Université d'Orléans) and negligible in summer. This karstic hydrologic system is the largest in France in terms of flow (tens to hundreds of $\mathrm{m}^{3} / \mathrm{s}$ ) and provides the main water resource of the city of Orléans. Chemical compositions of influent waters (River Loire) and effluent waters (spring of the river Loiret) were compared, in particular during floods in summer 1992 and 1993 (Fig. 2, Fig. 3 and Fig. 4). Variation of chloride in the River Loire during the stream rise can be used as an environmental tracer of the underground flow (Fig. 2). Short transit times of about 3 days are detectable (Fig. 2) which are consistent with earlier estimations obtained with chemical tracers (Ref. in Chéry, J.-L. (1983) Thèse, Université d'Orléans). Depending on the hydrological regime of the river, organic carbon discharge ranges between 3-7 and 2-13 mg/l for dissolved and particulate matter respectively (Fig. 3). Eutrophic characteristics and high algal biomasses are found in the River Loire during low water (Lair, N. and Sargos, D. (1993) A 10 year study at four sites of the middle course of the River Loire. I - Patterns of change in hydrological, physical and chemical variables in relation to algal biomass. Hydroécol. Appl. 5, 1-27) together with more organic carbon rich suspended particulate matter than during floods (30-40 $\mathrm{C}_{\text {org }} \%$ dry weight versus 5-10\%). Amounts of total organic carbon and dissolved oxygen (Fig. 3) dramatically decrease during the underground transport, whereas conversely, dissolved calcium, alkalinity and inorganic carbon increase (Fig. 4). Anoxia of outflows may start in April. Dissolution of calcium carbonates along the influent path outweighs closed system calcite equilibrium of inflow river waters (Table 3). The impact of organic matter oxidation on calcite dissolution may be traced by variations of alkalinity and total carbonates in water. Following, Jacobs, L. A., von Gunten, H. R., Keil, R. and Kuslys, M. (1988) Geochemical changes along a rivergroundwater infiltration flow path: Glattfelden, Switzerland. Geochim. Cosmochim. Acta52, 2693-2706), results are shown graphically (Fig. 5). Extent of reactions is controlled by the consumption of dissolved $\mathrm{O}_{2}$ and nitrate for organic matter oxidation and by the release of $\mathrm{Ca}^{2+}$ for calcite dissolution (Table 2). The karstic network is considered to behave like a biological reactor not exchanging with the atmosphere, with steady inhabitant microbial communities (Mariotti A., Landreau A. and Simon B. (1988) ${ }^{15} \mathrm{~N}$ isotope biogeochemistry and natural denitrification process in groundwater: Application to the chalk aquifer of northern France. Geochim. Cosmochim. Acta52, 1869-1878; Gounot, A.-M. (1991) Ecologie microbienne des eaux et des sédiments souterrains. Hydrogéologie, 239-248). Thus, energy requirements only are considered, not carbon assimilation. Moreover, there is no necessity to invoke any delay for nitrification enhancement, as observed elsewhere, after waste water discharge into the river (Chesterikoff, A., Garban, B., Billen, G. and Poulin, M. (1992) Inorganic nitrogen dynamics in the River Seine downstream from Paris (France).

Biogeochem. 17, 147-164). Main microbial processes are assumed to be aerobic respiration, nitrification and denitrification. Reactions with iron and manganese, real but not quantitatively important, were neglected. Sulphate reduction and methane formation, certainly not active, were not considered. Denitrification, which is suggested by low nitrate and ammonium concentrations and anoxia in the outflow, is known to be rapid enough to be achieved in a short time (Dupain, S. (1992) Dénitrification biologique hétérotrophe appliquée au traitement des eaux d'alimentation: Conditions de fonctionnement et mise au point d'un procédé. Thèse, Université Claude Bernard, Lyon). Reaction are somewhat arbitrary but conform to general acceptance (Morel, M. M. and Hering, J. G. (1993) Principles and Applications of Aquatic Chemistry. Wiley, New York). Anaerobic ammonium oxidation 
(Mulder A., van de Graaf, A. A., Robertson, L. A. and Kuenen, J. G. (1995) Anaerobic ammonium oxidation discovered in a denitrifying fluidized bed reactor. FEMS Microbiol. Ecol. 16, 177-184), although possible, was not considered. In fact, $\mathrm{C} / \mathrm{N}$ ratio of the reactive organic matter has only mild repercussions on the results; i.e. in the same range as the analytical errors for alkalinity and total carbonates. The objective was simply to roughly confront characteristics of outflowing waters and the calculation. Respective roles of aerobes and denitrifiers, for instance, are not certain. Several periods during low water or floods were selected with various ranges for calcium dissolution or nitrate and oxygen concentrations. The result is that in most cases simulation and data are in reasonable accordance (Fig. 5). Amounts of organic matter in River Loire are generally sufficient to sustain the process (Table 3).

Particulate organic matter is probably the most reactive. The balance of oxidation of organic matter indicates that about $65 \mu \mathrm{g} \mathrm{C}_{\text {org }} / 1 \cdot \mathrm{h}$ are oxidized during the transport without much variation with the river regime or organic discharge. It is concluded that limestone dissolution is directly dependent on organic matter oxidation, but variation occurs (7-29 $\left.\mathrm{mg} \mathrm{CaCO}_{3} / \mathrm{l}\right)$ with the level of bases that can be neutralized in the influent River Loire water.

\section{Mots-clé}

- fleuve Loire;

- source de Loiret;

- pertes fluviales;

- carbone organique;

- alccalinité;

- calcaires;

- dissolution;

- anoxie;

- ré surgences;

- aquifè re karstique

\section{Mots-clé}

- river Loire;

- $\quad$ spring of the river Loiret;

- influent water;

- stream sink;

- organic carbon;

- alkalinity;

- limestone dissolution;

- anoxic outflow;

- karst aquifer

\section{Introduction}

Les aquifères rechargés directement au contact d'une masse d'eau (lac, rivière) peuvent voir évoluer leurs caractéristiques physico-chimiques en fonction de la quantité de matière organique qui s'y introduit. Ce phénomène s'observe à proximité immédiate des zones de recharge des aquifères alluviaux (Jacobs et al., 1988; Von Gunten et al., 1991; Bourg and Bertin, 1993). Le même type d'alimentation appliqué au cas des aquifères karstiques permet d'envisager l'extension du phénomène sur plusieurs dizaines de kilomètres. Dans le val d'Orléans, l'aquifère karstique des calcaires de Beauce (qui constitue la principale ressource 
en eau de la ville) est alimenté en majeure partie par des pertes du fleuve Loire (Zunino et al., 1980). La variation saisonnière du débit et du chimisme de l'eau aux exutoires indique l'influence du fleuve sur l'aquifère qui est de plus le siège de réactions particulières responsables du déficit en oxygène et de l'enrichissement en calcium des eaux résurgentes (Chéry, 1983; Livrozet, 1984). Le phénomène concerne directement la dissolution et l'érosion des calcaires, mais aussi, le cas échéant, la mobilisation des polluants métalliques ou organiques (Schwarzenbach et al., 1983; Sigg et al., 1992).

On a cherché à simuler le changement de composition chimique des eaux entre l'entrée et la sortie du système karstique par le jeu de différentes réactions impliquant l'oxydation de la matière organique. Le bilan de ces réactions a été calculé pour des conditions hydrologiques différentes, en suivant les crues amorcées à Orléans le 4 juin 1992 et le 15 septembre 1993.

\section{Matériel et méthodes}

\subsection{Hydrogéologie du système karstique du Val d'Orléans}

L'enfoncement de la Loire au cours du Pléistocène supérieur a entraîné, dans le Val d'Orléans, l'érosion de la formation peu perméable de Sologne (Burdigalien) et la création d'un réseau de drainage karstique dans les calcaires lacustres de la formation de Beauce sous-jacente. La démonstration par traçage de l'alimentation abondante du système karstique par les pertes du fleuve a été apportée dès le début du siècle par plusieurs études (références dans Chéry, 1983) qui ont démontré l'existence de relations directes et rapides (environ $150 \mathrm{~m} / \mathrm{h}$ ) entre les pertes du fleuve situées en amont du val, et les captages et les résurgences naturelles (sources du Loiret et de la Pie) situés 10 à $20 \mathrm{~km}$ en aval (Fig. 1). Les bilans hydrologiques annuels dans le Val d'Orléans indiquent que le fleuve contribue à plus de $80 \%$ à l'alimentation du système aquifère (Zunino et al., 1980; Chéry, 1983; Livrozet, 1984). L'alimentation comprend des pertes massives (dont les manifestations sont observables épisodiquement) et probablement des infiltrations diffuses à travers les alluvions, l'ensemble formant, par le débit, le système karstique majeur de France (les débits de pertes varieraient de quelques $\mathrm{m}^{3} / \mathrm{s}$ en période d'étiage à plusieurs centaines de $\mathrm{m}^{3}$ /s en période de crue; Chéry, 1983, Zunino et al., 1980). 


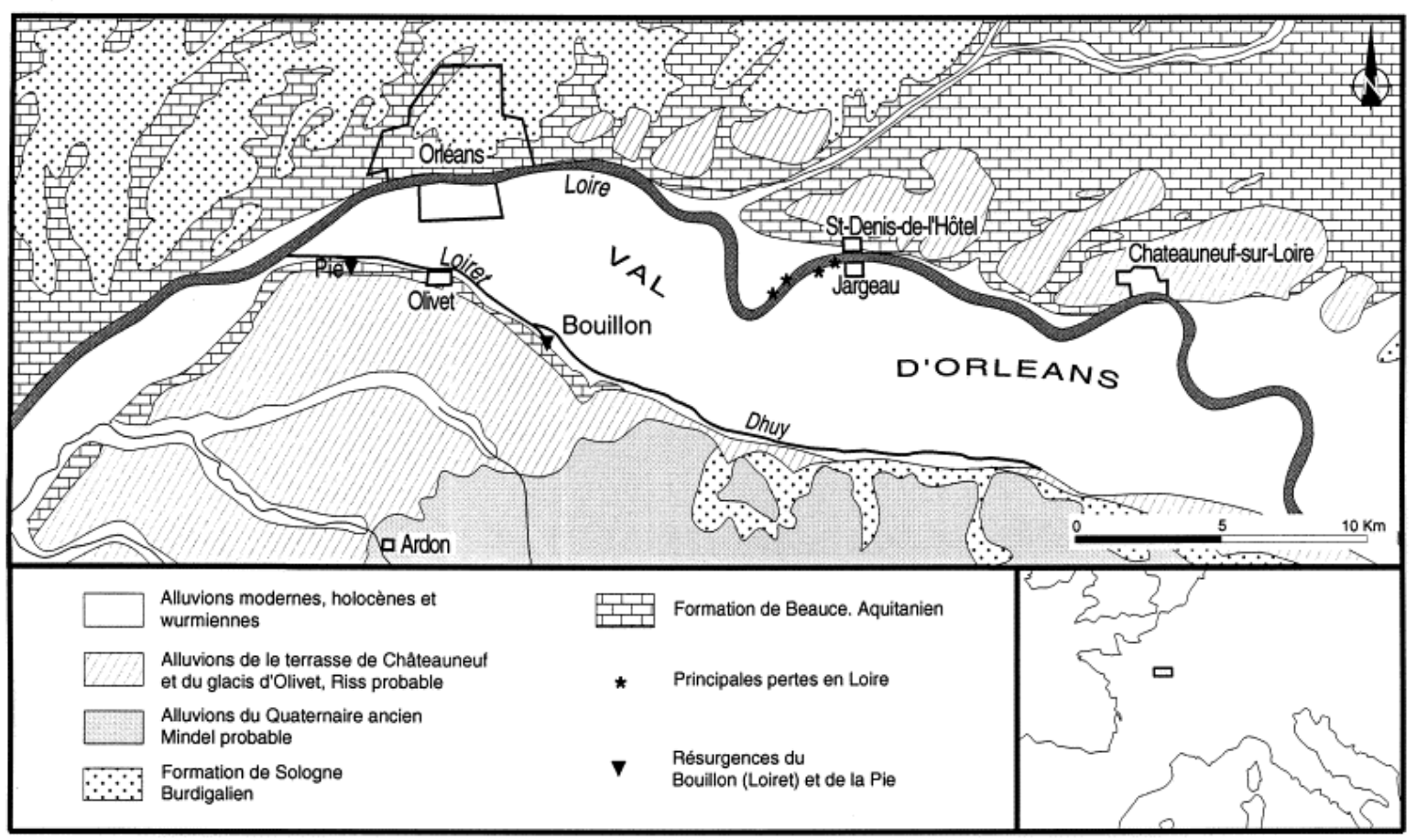

Fig. 1. Situation géographique du système. Les principales zones de pertes en amont d'Orléans $\left({ }^{*}\right)$ ainsi que les principales résurgences $(\nabla)$ ont été figurées, en particulier la source du Loiret, dite du "Bouillon".Fig. 1. Study area location with sites of the main stream sinks $\left({ }^{*}\right)$ and resurgences $(\nabla)$, particularly the spring of the River Loiret, the so-called "Bouillon".

La source du Loiret dite du "Bouillon" (Fig. 1), apparemment isolée du cours du Dhuy (rivière alimentée par les eaux de ruissellement de l'impluvium local) au XVIIeme siècle (Boitel, 1965), constitue un point d'observation privilégié des sorties du système (Chéry, 1983; Livrozet, 1984), à nouveau utilisé pour cette étude. Le débit moyen de la source est de l'ordre d'un $\mathrm{m}^{3} / \mathrm{s}$, il ne représente qu'une partie du flux transitant à travers le système, de nombreuses sorties existent le long du Loiret, de la Pie et du cours même de la Loire à l'ouest d'Orléans. Les débits à la source du Bouillon varient entre 0,3 et $3 \mathrm{~m}^{3} / \mathrm{s}$ et sont assez bien corrélés à ceux de la Loire dont le débit moyen d'étiage est voisin de $80 \mathrm{~m}^{3} / \mathrm{s}$ avec des crues en saison froide dépassant souvent $1500 \mathrm{~m}^{3} / \mathrm{s}$ (Chéry, 1983).

\subsection{Situation climatique et hydrologique des périodes étudiées}

La saison estivale, en raison de l'importance minimale des infiltrations de la zone insaturée vers l'aquifère, est particulièrement propice au suivi des relations Loire-résurgences. En hiver, au contraire, une partie des différences entre le chimisme des eaux du fleuve et de la source du Bouillon peut être attribuée à la contribution d'eaux provenant de l'impluvium local (résultats non publiés). Par ailleurs, la variation rapide du chimisme des eaux, provoquée par les crues du fleuve, offre la possibilité d'appréhender les relations entre les masses d'eaux et d'évaluer le temps de transit. 
Deux épisodes de crue en période estivale sont donc présentés ici. La crue amorcée le 3 juin 1992 à Orléans, consécutive à des précipitations abondantes tombées sur le Massif Central, faisait suite à une longue période d'étiage commencée vers le 15 avril avec sur le plan local des précipitations faibles (moins de $50 \mathrm{~mm} /$ mois en avril et mai, soit la moitié des précipitations normales). Des niveaux élevés et fluctuants se sont maintenus en Loire jusqu'à la mi-juillet, pour laisser place ensuite aux bas niveaux jusqu'au 24 septembre, début d'une nouvelle période de montée du niveau de l'eau (Fig. 2). La période étudiée en 1993 correspond à la première remontée du niveau de l'eau après l'étiage estival et complète ainsi l'étude de l'année précédente (Fig. 2).

1992
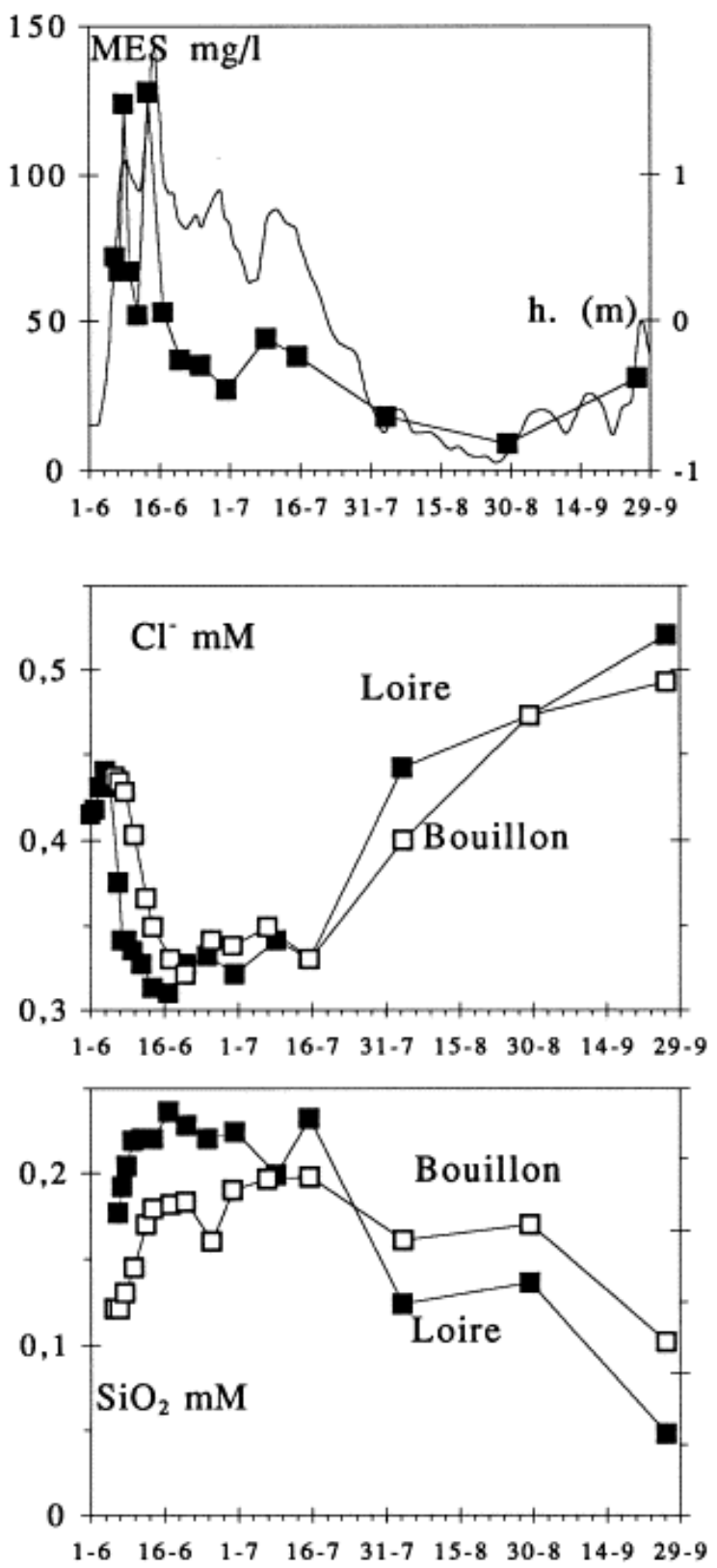

1993
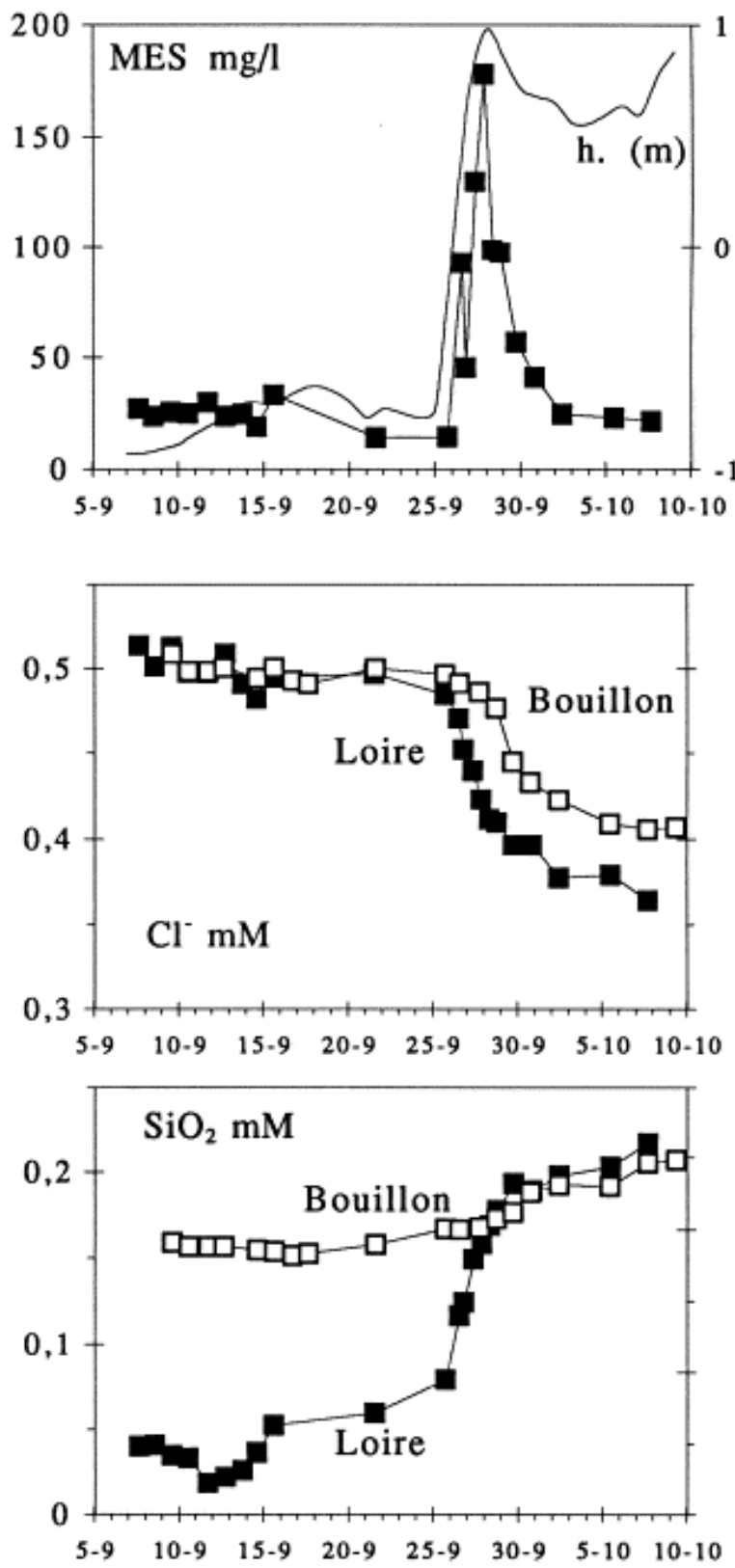

Fig. 2. Hauteurs d'eau (échelle d'Orléans, cotes entre -1 et $2 \mathrm{~m}$, ligne continue) et matières en suspension (MES en $\mathrm{mg} / \mathrm{l}$, carrés noirs) en Loire pendant les deux périodes considérées 
(correspondance approximative hauteur/débit: $+1 \mathrm{~m}: 700 \mathrm{~m}^{3} / \mathrm{s} ; 0 \mathrm{~m}: 250 \mathrm{~m}^{3} / \mathrm{s} ;-1 \mathrm{~m}: 70 \mathrm{~m}^{3} / \mathrm{s}$ ). Chlorure et silice dissoute (mM) en Loire (carrés noirs) et à la source du Bouillon (carrés blancs). Noter qu'une subdivision des abscisses vaut 3 jours pour la période 1992 et 1 jour seulement pour la période 1993.Fig. 2. River Loire water level at Orléans in metres (solid line) and particulate matter (MES in mg/l; black squares). Rough water level/discharge relation: $+1 \mathrm{m:} 700 \mathrm{~m}^{3} / \mathrm{s} ; 0 \mathrm{m:}: 250 \mathrm{~m}^{3} / \mathrm{s}$; $-1 \mathrm{~m}: 70 \mathrm{~m}^{3} / \mathrm{s}$. Chloride and silica (mM) in the River Loire (black squares) and the spring of the Loiret; "Bouillon" (white squares). One minor division is equal to 3 days in 1992 and 1 day only in 1993

\subsection{Prélèvements et analyses}

Les prélèvements en Loire ont été effectués depuis les berges, d'abord à Jargeau (15 km en amont d'Orléans) où sont connues les principales pertes (Fig. 1), puis à Orléans, aucun facteur n'étant susceptible d'entraîner des différences importantes entre les deux sites (Chéry, 1983). Les eaux résurgentes ont été prélevées à la source du Bouillon (source du Loiret située dans l'enceinte du Parc Floral de la ville d'Orléans). Les eaux ont été filtrées sur filtre en fibre de verre (Wattman $\mathrm{GF} / \mathrm{F})$ ou sur filtre membrane $(0,45 \mu \mathrm{m})$.

Le Table 1 récapitule, pour chaque élément, la méthode d'analyse utilisée.

Table 1. Methodes d'analyseTable 1. Analytical methods

\section{Elément/paramètre}

$\mathrm{O}_{2}$

$\mathrm{pH}$

Alcalinité

Silice

$\mathrm{Cl}^{-}$

$\mathrm{NO}_{3}{ }^{-}$

$\mathrm{Na}^{+}, \mathrm{K}^{+}, \mathrm{Mg}^{2+}$

$\mathrm{Ca}^{2+}$

COD

COP

$\mathrm{C} / \mathrm{N}$

Fraction

hydrocarbonée

\section{Méthode d'analyse}

sonde à oxygène

électrode $\mathrm{pH}$

titration de Gran

colorimétrie (complexe molybdique réduit)

colorimétrie, électrophorèse capillaire (EC)

absorbance UV, EC

absorption atomique, $\mathrm{EC}$

titrimétrie, EC oxydation catalytique (TCM Carlo Erba)

combustion (Carmhögraph)

CHN Carlo Erba

pyrolyse Rock-Eval (Espitalié et al., 1985)

\section{Précision minimale}

$10 \%$

$\pm 0,1$

$2 \%$

$5 \%$

$5-10 \%$

$5-10 \%$

$10 \%$

$10 \%$

$10 \%$

$10 \%$

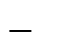

COD: $\mathrm{C}$ organique dissous. COP: $\mathrm{C}$ organique particulaire.

La somme des carbonates dissous $\left(\mathrm{C}_{\mathrm{T}}\right)$ a été déduite des mesures de $\mathrm{pH}$ et d'alcalinité (corrigées des teneurs en silice). Les constantes d'équilibre ont été calculées selon les relations tirées de WATEQ(B) (d'après Plummer et al., 1976), et corrigées en fonction de la force ionique par la relation de Güntelberg. Force ionique et température moyenne typiques des 
eaux en période estivale valent respectivement 0,003 et $20^{\circ} \mathrm{C}$ en Loire et 0,004 et $15^{\circ} \mathrm{C}$ à la source du Bouillon.

\subsection{Modèle stoechiométrique}

L'objectif est de simuler la modification de la composition chimique de l'eau au cours de son transit souterrain. Les réactions prises en compte sont l'oxydation de la matière organique (MO) et la dissolution du carbonate de calcium. Les variations de teneurs en $\mathrm{O}_{2}$ et $\mathrm{NO}_{3}{ }^{-}$ servent d'indicateurs de l'extension des réactions d'oxydo-réduction dont l'incidence sur l'alcalinité et la somme des carbonates est représentée graphiquement (Jacobs et al., 1988). Chaque réaction est représentée par un segment de droite dont la pente dépend des coefficients stoechiométriques et la longueur de la valeur de l'indicateur d'extension (Fig. 5). L'incidence des différentes réactions est explicitée Table 2.

Table 2. Réactions utiles au calcul de l'impact de l'oxydation de la matière organique sur la composition chimique de l'eau en transit. Les facteurs indiqués dépendent de l'extension des réactions et correspondent aux vecteurs du diagramme $\mathrm{Alc} / \mathrm{C}_{\mathrm{T}}$ (Fig. 5)Table 2. Reactions used to calculate the impact of the oxidation of organic matter on the chemical composition of the flowing waters. Vectors in the Alc/CT diagram (Fig. 5 ) are expressed in term of the extent indicators for each reaction

$\begin{array}{cc}\text { Facteur } & \text { Facteur } \\ \text { incident sur } & \text { incident sur } \\ \mathbf{C}_{\mathrm{T}} & \text { Alc }\end{array}$

(1) Aérobiose+nitrification ${ }^{\mathrm{a}}$

(1a) $4 / 5\left\{\left(\mathrm{CH}_{2} \mathrm{O}\right)+\mathrm{O}_{2} \rightarrow \mathrm{H}^{+}+\mathrm{HCO}_{3}^{-}\right\}$

(1b) $1 / 5\left\{1 / 2\left(\mathrm{NH}_{3}\right)+\mathrm{O}_{2} \rightarrow 1 / 2 \mathrm{H}^{+}+1 / 2 \mathrm{H}_{2} \mathrm{O}+1 / 2 \mathrm{NO}_{3}{ }^{-}\right\} \quad+\frac{4}{5}\left|\Delta \mathrm{O}_{2}\right| \quad \frac{1}{10}\left|\Delta \mathrm{O}_{2}\right|$

(2) Dénitrification ${ }^{\mathrm{a}}$

$5 / 4\left(\mathrm{CH}_{2} \mathrm{O}\right)+\mathrm{NO}_{3}{ }^{-} \rightarrow 1 / 4 \mathrm{H}^{+}+1 / 2 \mathrm{H}_{2} \mathrm{O}+5 / 4 \mathrm{HCO}_{3}{ }^{-}+1 / 2 \mathrm{~N}_{2}+\frac{5}{4} \mathrm{QNO}_{3}{ }^{-}+\mathrm{QNO}_{3}{ }^{-}$

(3) Dissolution ${ }^{b}$

$\mathrm{CaCO}_{3(\mathrm{~s})}+\mathrm{H}^{+} \rightarrow \mathrm{Ca}^{2+}+\mathrm{HCO}_{3}{ }^{-} \quad+\Delta \mathrm{Ca}^{2+} \quad+2 \Delta \mathrm{Ca}^{2+}$

$\mathrm{C}_{\mathrm{T}}=\left[\mathrm{H}_{2} \mathrm{CO}_{3}\right]+\left[\mathrm{HCO}_{3}{ }^{-}\right]+\left[\mathrm{CO}_{3}{ }^{2-}\right]$ Alcalinité

$(\mathrm{Alc})=\left[\mathrm{HCO}_{3}{ }^{-}\right]+2\left[\mathrm{CO}_{3}{ }^{2-}\right]+\left[\mathrm{H}_{3} \mathrm{SiO}_{4}{ }^{-}\right]+\left[\mathrm{OH}^{-}\right]-\left[\mathrm{H}^{+}\right]$Réserve alcaline

$\left(\mathrm{R}_{\mathrm{B}}\right)=2\left[\mathrm{Ca}^{2+}\right]+2\left[\mathrm{Mg}^{2+}\right]+\left[\mathrm{Na}^{+}\right]+\left[\mathrm{K}^{+}\right]-2\left[\mathrm{SO}_{4}{ }^{2-}\right]-\left[\mathrm{Cl}^{-}\right]-\left[\mathrm{NO}_{3}{ }^{-}\right] \Delta X=[X]_{\text {Bouillon }}-[X]_{\text {Loire }} \mathrm{QN}$

$\mathrm{O}_{3}{ }^{-}=1 / 10\left|\Delta \mathrm{O}_{2}\right|-\Delta \mathrm{NO}_{3}{ }^{-} \cdot \mathrm{QNO}_{3}{ }^{-}$représente la quantité de nitrate participant à la

réaction de dénitrification. ${ }^{a}$ Les réactions ont été écrites pour une mole d'accepteur

d'électrons.Pour l'étape aérobie, la composition théorique de la matière organique a été fixée avec un rapport $\mathrm{C} / \mathrm{N}$ égal à $8\left\{\left(\mathrm{CH}_{2} \mathrm{O}\right)_{8}\left(\mathrm{NH}_{3}\right)\right\}$. La réduction du carbone minéral par les bactéries nitrifiantes n'a pas à être considérée ici puisqu'elle est compensée par l'extension de la réaction 1a. Pour le processus de dénitrification, seule la fraction carbonée de la matière organique a été considérée. ${ }^{b}$ De manière alternative, l'extension de la réaction de dissolution pourrait être représentée par les quantités de protons produites aux réactions (1) et (2) à condition d'en soustraire les différences de 
concentrations observées entre la source du Bouillon et la Loire pour $\mathrm{OH}^{-}, \mathrm{CO}_{3}{ }^{2-}$, $\mathrm{H}_{3} \mathrm{SiO}_{4}{ }^{-}, \mathrm{H}_{2} \mathrm{CO}_{3}$ et $\mathrm{H}^{+}$. En effet, la variation de réserve alcaline associée aux 3 réactions peut s'écrire $\Delta \mathrm{R}_{\mathrm{B}}=-1 / 10\left|\Delta \mathrm{O}_{2}\right|+\mathrm{QNO}_{3}{ }^{-}+2 \Delta \mathrm{Ca}^{2+}$ avec

$\Delta \mathrm{R}_{\mathrm{B}}=\Delta \mathrm{Alc}=\Delta \mathrm{HCO}_{3}{ }^{-}+2 \Delta \mathrm{CO}_{3}{ }^{2-}+\Delta \mathrm{H}_{3} \mathrm{SiO}_{4}{ }^{-}+\Delta \mathrm{OH}^{-}-\Delta \mathrm{H}^{+}$. De même, $\Delta \mathrm{C}_{\mathrm{T}}=4 / 5\left|\Delta \mathrm{O}_{2}\right|+5 / 4 \mathrm{QNO}_{3}{ }^{-}+\Delta \mathrm{Ca}^{2+}=\Delta \mathrm{H}_{2} \mathrm{CO}_{3}+\Delta \mathrm{HCO}_{3}{ }^{-}+\Delta \mathrm{CO}_{3}{ }^{2-}$. En combinant les deux équations, il vient:

$\Delta \mathrm{Ca}^{2+}=9 / 10\left|\Delta \mathrm{O}_{2}\right|+1 / 4 \mathrm{QNO}_{3}{ }^{-}+\Delta \mathrm{CO}_{3}{ }^{2-}+\Delta \mathrm{OH}^{-}+\Delta \mathrm{H}_{3} \mathrm{SiO}_{4}{ }^{-}-\Delta \mathrm{H}^{+}-\Delta \mathrm{H}_{2} \mathrm{CO}_{3}$, ce qui est équivalent à la quantité de protons libérée par les réactions (1) et (2) moins la quantité nécessaire à la balance des espèces réactives.Le calcul s'applique parfaitement à la comparaison des eaux de Loire et de la source du Bouillon qui présentent, en période estivale, des variations de réserve alcaline dues essentiellement aux variations des teneurs en calcium et nitrate.

L'écriture des réactions est en partie arbitraire, mais est conforme à la présentation courante (Stum and Morgan, 1981; Morel and Hering, 1993). Une limitation propre au site étudié est le temps de réaction relativement court (environ 3 jours) dans l'eau en transit. Le développement de certains processus bactériens, nitrification notamment, pourrait nécessiter un délai plus important (exemple des rejets de station d'épuration en Seine, Chesterikoff et al., 1992). Dans le cas étudié, il convient de considérer une biomasse microbienne résidante dans le milieu souterrain et non obligatoirement observable dans les eaux circulantes (Mariotti et al., 1988; Gounot, 1991). Les processus microbiens peuvent ainsi s'établir malgré le transit rapide de l'eau, et même être entretenus grâce au renouvellement rapide du milieu (accepteurs d'électrons, notamment). En ce qui concerne la MO, il semble que la fraction particulaire joue un rôle prépondérant (voir discussion). Le temps de transit des particules dans le système karstique étant probablement supérieur à celui de l'eau, les étapes de dégradation de la MO les moins rapides (dépolymérisation, hydrolyse) peuvent se dérouler avec un délai supérieur à 3 jours. Cela soulève la question du stockage souterrain temporaire du carbone organique (voir discussion). Les quantités de carbone organique mesurées en Loire ne sont pas utilisées directement dans le calcul, il suffit que pendant la période considérée, les entrées potentielles par les pertes massives dans le lit du fleuve (par des ouverture dans le calcaire et à travers les alluvions grossiers) soient au moins égales aux quantités oxydées en profondeur (voir discussion et Table 3). L'extension des réactions ne présume pas non plus l'état d'équilibre des solutions vis à vis de la calcite. 
Table 3. Différence Loire-Bouillon pour $\mathrm{C}_{\text {org }}$ et $\mathrm{Ca}^{2+}$ : observations et calculTable 3 . Differences in $\mathrm{C}_{\text {org }}$ and $\mathrm{Ca}^{2+}$ between the River Loire and the outflow (Bouillon): field data versus calculation

Périodes

$$
\mathrm{C}_{\text {org }}(\mathrm{mmol} / \mathrm{l}) \quad \mathrm{Ca}^{2+}(\mathrm{mmol} / \mathrm{l})
$$

$$
\text { COP (1) } \left.\left.{ }^{\mathrm{a}} \text { COD (1) }\right)^{\mathrm{a}} \text { Corg oxydé (2) (3) }\right)^{\mathrm{a}} \quad(4)^{\mathrm{b}}
$$

1992

$\begin{array}{llllll}\text { 8-10 juin } & 0,40 & 0,17 & 0,32 & 0,25 & 0,08 \\ \text { 1-16 juillet } & 0,25 & 0,08 & 0,31 & 0,25 & 0,0 \\ \text { 23 août } & 0,42 & 0,04 & 0,17 & 0,22 & -0,14 \\ \text { 29 août } & 0,29 & 0,08 & 0,18 & 0,19 & -0,10 \\ 1993 & & & & & \\ \text { 9-11 septembre } & 0,67 & 0,08 & 0,43 & 0,18 & -0,23 \\ \text { 14-20 septembre } 0,42 & 0,13 & 0,43 & 0,07 & -0,25 \\ \text { 25-26 septembre } 1,00 & 0,08 & 0,30 & 0,19 & -0,05 \\ \text { 28-30 septembre } 0,58 & 0,17 & 0,36 & 0,24 & 0,08 \\ \text { 1-5 octobre } & 0,17 & 0,04 & 0,38 & 0,29 & 0,07\end{array}$

(1) COP et COD (diminution observée entre la Loire et la source du Bouillon). (2) $\mathrm{C}_{\mathrm{org}}$ oxydé (calculé suivant les réactions 1 and 2 Table 2). (3) Calcium dissous (quantités observées). (4) Calcium dissous (ou précipité, pour les valeurs négatives) dans l'hypothèse d'une simple équilibration des eaux de Loire avec la calcite en milieu fermé sans oxydation de matière organique (calculé de manière itérative en considérant constant le terme " $\mathrm{R}_{\mathrm{BLoire}}-\mathrm{Ca}^{2+}$ "; Michard, 1989). On constate: (i) que les quantités de $\mathrm{C}$ organique contenues en Loire sont en général suffisantes pour entretenir les réactions envisagées; (ii) que les quantités de $\mathrm{Ca}^{2+}$ dissous dans le système sont supérieures à la capacité de dissolution des eaux de Loire. ${ }^{\mathrm{a}}$ Différence mesurée entre Loire et Bouillon (avec un décalage de 3 jours pour le Bouillon). ${ }^{b}$ L'erreur sur ces valeurs calculées peut atteindre $30 \%$ (essentiellement à cause de l'incertitude sur le $\mathrm{pH}$ ).

Les très faibles teneurs en nitrate et ammonium à la résurgence en période estivale (Chéry, 1983; Livrozet, 1984), suggèrent l'existence d'un mécanisme de dénitrification que l'anoxie des eaux résurgentes et la rapidité du processus hétérotrophe (Dupain, 1992) rendent plausible, d'autant plus que le manque d'apport organique constaté habituellement dans les aquifères (Mariotti, 1986) est ici pallié par les entrées d'eau de Loire. La dénitrification basée sur l'oxydation anaérobie des ions ammonium (récemment mise en évidence en station d'épuration, Mulder et al., 1995) n'a pas été prise en compte, ce qui explique le choix de la formule utilisée pour la MO à l'étape anoxique (réaction 2, Table 2), différente de celle de la phase aérobie (réaction 1, Table 1) qui comprend le processus de nitrification. L'incertitude concernant la proportion d'azote dans la matière organique réagissante a d'ailleurs peu de 
répercussion sur les résultats finaux de la simulation (moins de $20 \mu \mathrm{mol}$ sur les valeurs calculées de $\mathrm{C}_{\mathrm{T}}$ et Alc).

Etant données les concentrations mises en jeu, les autres accepteurs d'électrons (oxydes de manganèse ou de fer, sulfate) et la méthanogenèse $\left(\mathrm{CH}_{4}<0,1 \mathrm{mg} / \mathrm{l}\right)$ peuvent être négligés (Chéry, 1983; Livrozet, 1984; cette étude).

En ce qui concerne la dissolution du calcaire (réaction 3, Table 2), l'extension de la réaction dépend d'une partie seulement des protons libérés par les deux premières réactions, l'autre partie étant nécessaire pour compenser les différences de concentrations des espèces réactives d'un point de vue acido-basique entre les eaux de Loire et celles du Bouillon. La variation de réserve alcaline entre Loire et Bouillon dépendant essentiellement de celle de $\mathrm{Ca}^{2+}$ et $\mathrm{NO}$, l'extension de la réaction de dissolution peut indifféremment (et plus simplement) être indiquée par la variation du calcium (voir Table 2).

\section{Resultats - discussion}

Les résultats des analyses sont représentés graphiquement Fig. 2, Fig. 3 and Fig. 4. Les variations de régime du fleuve sont représentées Fig. 2 par les hauteurs d'eau en Loire et les teneurs en matière en suspension (MES). A la source du Bouillon il n'existe pas de relation entre les débits et les hauteurs d'eau en raison du manque de pente et de la présence en aval de nombreux biefs et barrages (Chéry, 1983). Les relations entre les débits de la Loire et de la source du Bouillon ont été décrites antérieurement (Chéry, 1983). 

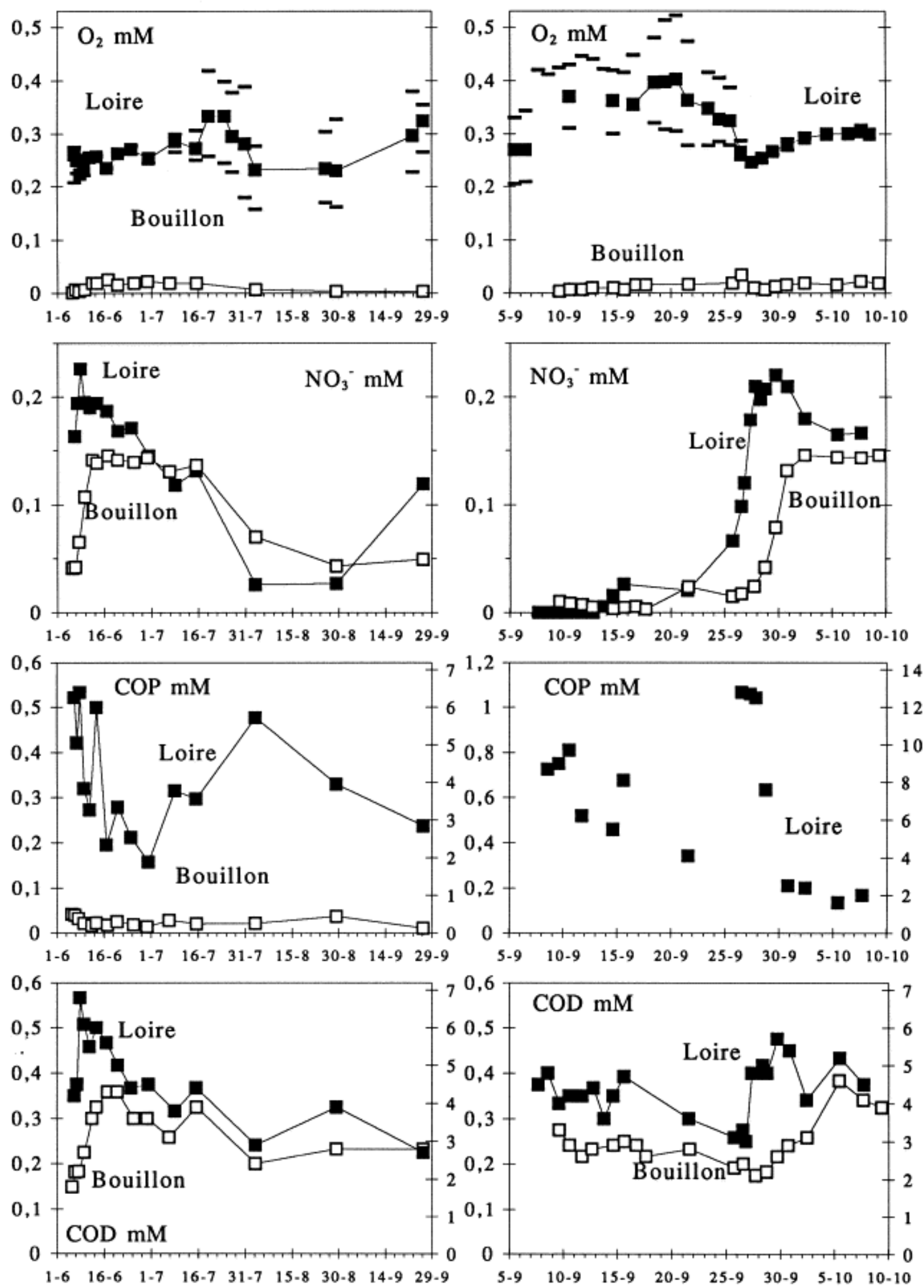

Fig. 3. Oxygène dissous, nitrate, carbone organique particulaire (COP) et carbone organique dissous (COD) en mM, en Loire et à la source du Bouillon. Pour l'oxygène dissous en Loire, les valeurs 
journalières moyennes $(n=4)$ et les valeurs extrêmes ont été indiquées (données incluant les mesures faites à la station d'alerte de St Denis de l'Hôtel). Pour COP et COD, une échelle en $\mathrm{mg} / \mathrm{l}$ est également indiquée (graduation du côté droit des graphes).Fig. 3. Dissolved oxygen, nitrate, POC and DOC (mM) in the River Loire and Bouillon spring waters. Diel variation (mean and extreme values) for dissolved oxygen is indicated. 

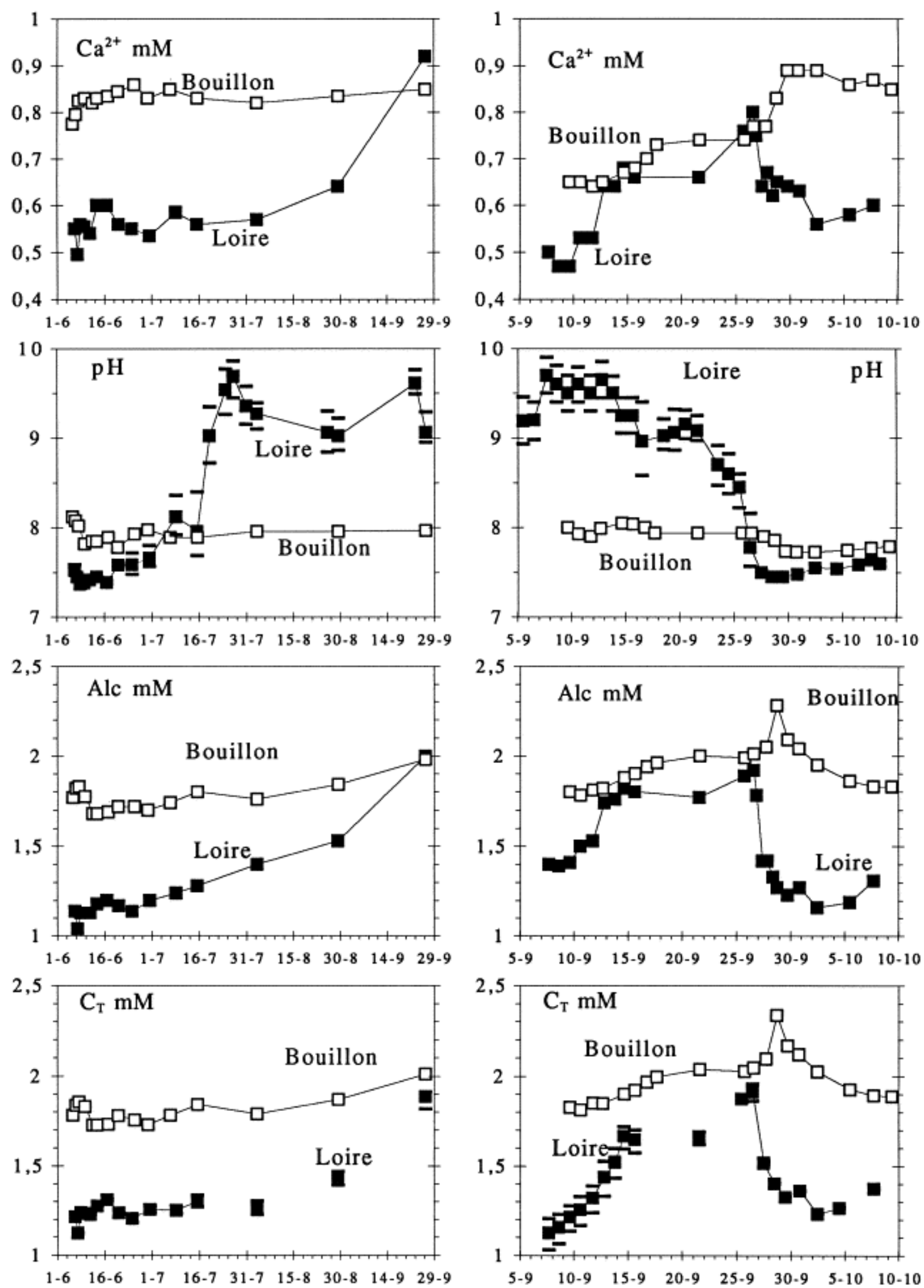

Fig. 4. Calcium, pH, alcalinité (Alc) et somme des carbonates $\left(C_{T}\right)$. Comme pour l'oxygène dissous (Fig. 3), la variation nycthémérale du pH en Loire a été représentée par la valeur moyenne et les valeurs 
extrêmes. L'incidence sur la valeur calculée de $C_{T}$ est représentée de la même façon.Fig. 4. Calcium, $\mathrm{pH}$, alkalinity (Alc) and total inorganic carbon $\left(\mathrm{C}_{\mathrm{T}}\right)$. Diel variation in River Loire is reported for $\mathrm{pH}$ as well as for $\mathrm{C}_{\mathrm{T}}$.

\subsection{Chlorure et silice (Fig. 2)}

Les teneurs en chlorure (ainsi qu'en sodium et lithium, résultats non figurés) diminuent brutalement dans les eaux de Loire au cours des deux épisodes de crue (Fig. 2). L'impulsion initiale de la variation est répercutée à la résurgence avec un déphasage d'environ 3 jours (c'est à dire une subdivision de l'abscisse du graphique en 1992; 3 subdivisions pour 1993). Les eaux résurgentes n'atteignent cependant le pallier inférieur observé en Loire qu'après un délai plus long, qui traduit probablement le trajet plus long de certaines fractions de la tranche d'eau et donc un certain mélange pendant le transit. La silice, malgré une réactivité plus importante que les chlorures pendant le transit (dissolution en période d'étiage et d'eutrophisation; apparente insolubilisation pendant les hauts niveaux d'eau), ainsi que les nitrates (Fig. 3), permettent également de déterminer un décalage d'environ 3 jours entre les masses d'eau.

L'objectif étant d'étudier la variation de composition des eaux à travers le système hydrologique, les comparaisons doivent être faites à partir de valeurs caractéristiques mesurées sur une même tranche élémentaire du flux hydrique traversant le système. Compte tenu de la bonne concordance entre les observations antérieures, fondées sur l'interprétation des traçages artificiels (Chéry, 1983; Livrozet, 1984) et celles déduites du suivi des épisodes de crue, on considérera pour la suite, que les eaux du Bouillon correspondent, pour l'essentiel, à la résurgence des pertes de Loire après 3 jours de transit souterrain.

\subsection{Oxygène dissous, nitrate et carbone organique (Fig. 3)}

En période estivale, les teneurs en oxygène dissous des eaux de Loire peuvent subir de fortes variations nycthémérales qui s'atténuent au moment des crues. Les valeurs moyennes et l'amplitude de la variation sont présentées Fig. 3 (données incluant des mesures de la station d'observation de la Loire à St Denis de l'Hôtel). On note des valeurs sensiblement supérieures pendant la seconde période étudiée (septembre 1993). Pendant les périodes considérées, la teneur en oxygène dissous des eaux du Bouillon demeure très faible $(<0,6 \mathrm{mg} / \mathrm{l}$ soit $0,02 \mathrm{mM}$ ). Une très légère remontée est perceptible au moment des crues (Fig. 3) sans cependant atteindre les teneurs relativement élevées (60\% de la saturation) mesurées en période hivernale (Chéry, 1983; Livrozet, 1984).

Les teneurs en nitrate augmentent avec les crues, aussi bien en Loire qu'au Bouillon, mais le palier maximum atteint à la résurgence est inférieur à celui atteint dans le fleuve, indiquant une possible consommation pendant le transit dans l'aquifère (Fig. 3). Pendant la période estivale, au contraire, les teneurs à la résurgence sont supérieures à celles présentes en Loire (proches de zéro).

Les épisodes de crue sont marqués également par l'augmentation des quantités de matières en suspension (MES; Fig. 2) et des teneurs en carbone organique (COP, COD; Fig. 3) qui décroissent rapidement (MES, COP) lorsque le niveau maximum des eaux est atteint. Malgré un taux de MES plus faible qu'en période de crue, les périodes de bas niveau des eaux 
présentent des concentrations élevées en COP qui résultent de fortes biomasses algales. La caractérisation détaillée des différents types de matières organiques fluviatiles n'est pas abordée ici bien que leurs potentiels métaboliques varient probablement en fonction de la teneur en fractions labiles (Ittekkot, 1988). Signalons cependant brièvement qu'à la couleur verte des particules algales des bas niveaux d'eau correspondent des fortes teneurs en $\mathrm{C}$ organique (30-40\%) et en produits de pyrolyse hydrocarbonés (500 à $800 \mathrm{mg} / \mathrm{g} \mathrm{C}_{\text {org }}$ distribués autour de $\left.300^{\circ} \mathrm{C}\right)$ qui les distinguent des particules brunes de crue $\left(\mathrm{C}_{\text {org }}: 5-10 \%\right.$; produits de pyrolyse: 270 à $350 \mathrm{mg} / \mathrm{g} \mathrm{C}_{\text {org }}$ centrés vers $400^{\circ} \mathrm{C}$ ). A l'inverse, les rapports $\mathrm{C} / \mathrm{N}$ (compris entre 7 et 10) ne distinguent pas les deux types de particules. La différence de comportement à la pyrolyse des deux ensembles d'échantillons tient certainement à la nature plus fraîche de la MO d'origine algale comparée à celle plus détritique des particules transportées par les crues (Scribe et al., 1995).

La teneur en COP à la source du Bouillon est constamment faible $(<0,5 \mathrm{mg} / \mathrm{l}$, Fig. 3$)$ mais, compte tenu de la très faible charge particulaire des eaux, la matière organique constitue certainement la fraction majeure des particules (avec un rapport $\mathrm{C} / \mathrm{N}$ bas; 6-7). Les teneurs en COD y sont comprises entre 2 et $4 \mathrm{mg} / \mathrm{l}(0,1-0,4 \mathrm{mM})$, et suivent celles mesurées en Loire, ce qui suggère le caractère inerte d'une partie importante du COD fluviatile (Fig. 3).

\subsection{Calcium, alcalinité, pH (Fig. 4)}

L'importance de la dissolution pendant le transit souterrain peut être appréciée par la différence de concentration en calcium entre Loire et Bouillon. Cette différence est maximale aux périodes de haut niveau des eaux (juin 1992, début octobre 1993), diminue légèrement au cours de l'été (1992) et est minimale à la fin de la période estivale lors de la toute première remontée du niveau d'eau (fin septembre 1992, mi-septembre 1993). La comparaison doit, bien entendu, tenir compte du décalage de 3 jours envisagé précédemment. Ainsi la valeur plus forte en Loire qu'au Bouillon mesurée fin septembre 1992 doit être interprétée en tenant compte de la brusque augmentation des teneurs consécutive au début de remontée des eaux en Loire, telle qu'on a pu l'observer en 1993.

La diminution plus marquée de l'alcalinité (=réserve alcaline, Tableau 2) que du calcium en Loire, suite à la crue de septembre 1993, correspond à l'établissement d'un rapport $\mathrm{Na} / \mathrm{Cl}$ plus faible qu'avant la crue $(1,2$ contre 1,4$)$ et à l'augmentation des nitrates.

Pendant la période d'étiage, on note pour le $\mathrm{pH}$ (comme pour l'oxygène dissous) des valeurs maximales en fin de journée et des valeurs minimales en fin de nuit (la moyenne et l'étendue de la variation, estimées en incluant les données de la station d'alerte de St Denis de l'Hôtel, sont représentées Fig. 4). Les valeurs calculées de la somme des carbonates dans les eaux de Loire $\left(\mathrm{C}_{\mathrm{T}}\right.$, Fig. 4) comportent par conséquent, elles aussi, une variation nycthémérale qui disparaît lors des épisodes de crue.

Le transit souterrain des eaux de Loire s'accompagnant de la dissolution d'une certaine quantité de calcaire, il semble approprié de décrire le degré de saturation des eaux entrantes en calculant les quantités de calcium qui se dissoudraient ou au contraire précipiteraient (en cas de sursaturation) lors du simple équilibre avec la calcite en système fermé. Le calcul par itération (Michard, 1989) montre (malgré l'imprécision due en particulier à la variation journalière du $\mathrm{pH}$ ) que sans l'intervention des réactions d'oxydation de la $\mathrm{MO}$, les eaux de Loire légèrement sous-saturées en période de crue ne pourraient dissoudre que quelques dizaines de micromoles par litre, tandis qu'à l'étiage, les eaux très sursaturées, en raison des 
phénomènes de photosynthèse, devraient laisser précipiter des quantités importantes de carbonates (Table 3).

\subsection{Définition des périodes caractéristiques et calcul des bilans}

L'objectif étant de tester la capacité des réactions d'oxydation de la MO à expliquer le changement de composition chimique des eaux entre la Loire et le Bouillon, des épisodes montrant des caractéristiques apparemment différentes ont été distingués. La description et l'analyse détaillées de chaque épisode ne sont pas envisagées, il s'agit simplement de permettre une appréciation générale de la validité du schéma proposé.

On retiendra en 1992: (1) la période de crue entre les 8 et 10 juin avec des teneurs en nitrate en Loire environ $60 \mu \mathrm{mol} / \mathrm{l}$ supérieures à celles observées dans les eaux du Bouillon 3 jours plus tard; (2) la période du 1 au 15 juillet marquée par le début de l'augmentation du pH en Loire avec des teneurs en nitrates égales en Loire et au Bouillon; (3) les périodes des 3 et 29 août qui correspondent aux bas niveaux des eaux en Loire avec des pH élevés, les teneurs en nitrates y sont plus faibles qu'à la source du Bouillon, les pics d' $\mathrm{O}_{2}$ en Loire sont importants mais les valeurs moyennes sont semblables à celles des périodes précédentes. La situation correspondant à la période fin septembre 1992 n'est pas décrite puisqu'on la retrouve mieux documentée en 1993, où on distinguera: (1) la période du 9 au 11 septembre qui traduit la fin de l'étiage estival avec des valeurs faibles des nitrates en Loire, des pH élevés et une dissolution encore marquée; (2) celle du 14 au 20 septembre, marquée par la forte augmentation de l'alcalinité et du calcium en Loire, sans nette répercussion au Bouillon, d'où une dissolution faible; (3) la période du 25-26 septembre qui correspond au début de la crue et aux pics de calcium et d'alcalinité en Loire ainsi qu'à la baisse des $\mathrm{pH}$ et à la reprise de la dissolution; (4) celle du 28 au 30 septembre, juste à la pointe de la crue, après la baisse de l'alcalinité et des teneurs en oxygène et en calcium en Loire, avec une différence importante des teneurs en nitrate et en calcium entre Loire et Bouillon; (5) enfin la période du 1 au 5 octobre qui fait suite à la montée des eaux et montre une légère remontée des teneurs en $\mathrm{O}_{2}$ en Loire, une faible différence des teneurs en nitrate et une dissolution importante.

La représentation graphique (Fig. 5) indique que les réactions envisagées décrivent de manière satisfaisante le changement de composition de l'eau au cours de son transit souterrain pour l'ensemble des périodes envisagées, y compris les périodes de forte eutrophisation, à condition de considérer les plus fortes valeurs déterminées pour $\mathrm{C}_{\mathrm{T}}$ en Loire (correspondant aux valeurs minimales du $\mathrm{pH}$ ). 

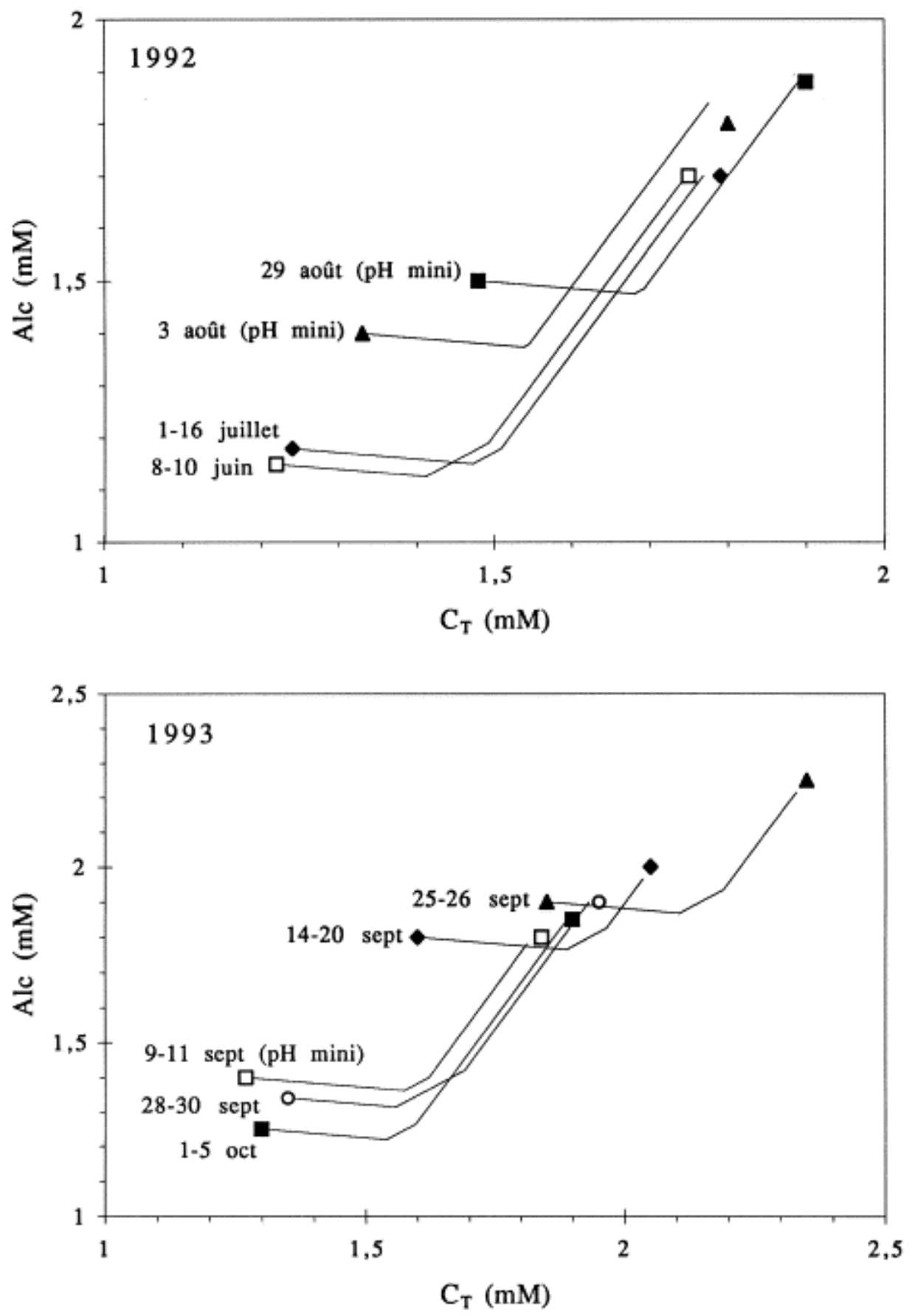

Fig. 5. Diagramme alcalinité/C minéral total (Jacobs et al., 1988). Pour chaque date, le point à gauche dans le diagramme correspond à la composition des eaux de Loire, le point de droite correspond aux eaux du Bouillon. A partir du point représentatif des eaux de Loire, le premier segment de droite traduit la modification de composition des eaux résultant des réactions d'oxydation aérobie de la matière organique (nitrification inclus, voir Table 2). Le deuxième segment correspond aux processus de dénitrification et le dernier à la dissolution du carbonate de calcium. Pour les périodes de forte eutrophisation (indiquées sur les diagrammes), les points représentatifs des eaux de Loire ont été figurés en considérant les $\mathrm{pH}$ minimum. La distance entre les points représentatifs des eaux résurgentes à la source du Bouillon et l'extrémité du dernier segment de droite permet d'apprécier l'écart entre les compositions mesurées et celles calculées.Fig. 5. Alkalinity versus inorganic carbon. 
For each date, the composition of the River Loire waters is shown by the symbol to the left of the diagram, whilst symbols on the right are for the Bouillon spring waters. From the River Loire symbol, the first straight line accounts for aerobic organic matter oxidation plus nitrification; the second for denitrification processes and the last for calcium carbonate dissolution. Minimum $\mathrm{pH}$ values were considered in the River Loire waters for eutrophic periods (as indicated on plots). Shift between Bouillon's symbols and end of segmented lines accounts for discrepancy between measured and computed water compositions.

Les quantités de carbone organique oxydé déduites du calcul (réactions 1 et 2 Fig. 5) varient presque d'un facteur $2(0,2-0,4 \mathrm{mM}$ soit $2-4 \mathrm{mg} / \mathrm{l}$; Table 3$)$ en fonction des quantités d'accepteurs d'électrons disponibles. Les valeurs les plus élevées correspondent aux périodes de plus forte teneur en oxygène dissous des eaux de Loire (fin de la période d'étiage en septembre 1993). La part de l'oxydation imputable aux processus anaérobies de dénitrification pourrait varier de 4 à 39\% suivant les périodes (bas niveaux et crues, respectivement). En fait, restreindre le rôle de la nitrification (et donc celui de la dénitrification) dans la simulation, n'a qu'une influence limitée (+10 à $30 \mu \mathrm{mol}$ au maximum) sur la quantité de $\mathrm{C}$ organique oxydé. L'impact sur l'alcalinité et la somme des carbonates est du même ordre de grandeur, qui est aussi celui de l'erreur analytique sur ces termes. Tel qu'il est envisagé, le jeu couplé des réactions intéressant l'azote fait que le choix du rapport $\mathrm{C} / \mathrm{N}$ de la matière organique réagissante n'a que peu de répercussion sur les résultats calculés. Le processus de dénitrification semble cependant bien réel pendant les périodes de crue si l'on considère le bilan des nitrates entre l'entrée et la sortie du système.

Compte tenu du contexte hydrogéologique du système étudié (transit rapide des eaux, existence de pertes massives, affleurement du substratum calcaire et d'alluvions grossiers dans le lit du fleuve), nous ne pensons pas que le siège des réactions envisagées puissent être principalement l'eau interstitielle des sédiments du lit du fleuve. En fait cette éventualité n'affecte en rien les résultats du schéma envisagé et ne peut donc pas relever de notre discussion. Compte tenu du temps de transit considéré, le taux d'oxydation moyen de la MO dans le milieu souterrain peut être estimé à environ $65 \mu \mathrm{g} \mathrm{C} / 1 \cdot \mathrm{h}$, la température moyenne des eaux étant respectivement 15 et $20^{\circ} \mathrm{C}$ pour le Bouillon et la Loire (période estivale). Pour comparaison, des taux compris entre 10 et $30 \mu \mathrm{g} \mathrm{C} / 1 \cdot \mathrm{h}$ peuvent être déduits des quantités d'oxygène consommé (in vitro à $20^{\circ} \mathrm{C}$ ) par oxydation bactérienne de $\mathrm{MO}$ dissoute d'eaux de Seine (Barillier, 1992). Ces taux sont évidemment très inférieurs (deux ordres de grandeur) à ceux entretenus dans les installations de traitement des eaux usées où plusieurs $\mathrm{mg} \mathrm{C/l} \cdot \mathrm{h}$ peuvent être oxydés (Czysz et al., 1989; Duchène, 1990).

\subsection{Relation avec le stock organique réel présent en Loire}

Malgré l'incertitude concernant l'importance respective des pertes massives (capables d'entraîner la totalité de la charge particulaire en suspension dans les eaux de Loire) et des pertes diffuses à travers les alluvions, les entrées potentielles de MO paraissent suffisantes pour assurer les réactions envisagées (Table 3). On note cependant qu'en fin de crue, lorsque la charge particulaire en suspension dans les eaux devient faible (Fig. 2 and Fig. 3), le niveau des entrées supposées est tout juste égal, voire inférieur, à la quantité nécessaire prévue par le calcul (période du 1 au 5 octobre 1993, par exemple). Cette observation suggère que le déficit 
en matière organique des entrées puisse être compensé par les excédants stockés précédemment. Cela revient à considérer que l'eau et la charge particulaire peuvent avoir un transit différent, comme cela a été évoqué plus haut pour justifier la prise en compte des processus de nitrification. En conséquence, la variation de la quantité de matière organique oxydée, dépendrait plus (à température égale) de la quantité disponible d'oxygène et de nitrate que du niveau des entrées en COP. Le compartiment COD semble moins impliqué dans le processus d'oxydation, comme le suggère le caractère fortement associé des variations au Bouillon et en Loire. Les périodes de crue (marquées par une forte augmentation du COD) montrent cependant une amplitude plus forte entre entrées et sorties. Cela pourrait s'expliquer par une plus grande biodégradabilité du stock dissous supplémentaire ou encore par sa plus forte affinité vis-à-vis des surfaces adsorbantes. La biodégradabilité du COD devrait être considérée (étude en projet) en rapport avec celle du COP d'origine détritique introduit dès le début de la crue, en remplacement du COP algal d'étiage plus riche en fractions métabolisables (et en produits pyrolysables, comme décrit plus haut).

\subsection{Comparaison avec les systèmes alimentés à travers une zone non saturée et rôle de l'oxydation de la MO fluviatile dans l'évolution du système karstique du Val d'Orléans}

Bien que la moyenne annuelle de la charge carbonatée dissoute acquise par les eaux de la Loire au cours de son transit souterrain soit faible $(35 \mathrm{mg} \mathrm{CaCO} / \mathrm{ll})$ comparée à celle exportée des systèmes karstiques alimentés, par les précipitations, à travers la zone non saturée (jusqu'à $300 \mathrm{mg} / \mathrm{l}$ ), les dissolutions spécifiques sont comparables, entre 50 et $75 \mathrm{~m}^{3} / \mathrm{km}^{2} /$ an (Chéry, 1983; Gonzalez, 1992; Chéry et al., 1982; Bakalowicz, 1979; Lepiller, 1980). Dans le cas du Val de Loire, la valeur élevée du débit compense la valeur relativement faible de la charge dissoute. Quelle que soit l'importance de la dissolution, l'évolution actuelle du réseau de drainage karstique relève aussi du colmatage, démontré par les sédiments rencontrés en forages ou en exploration spéléologique directe. L'évolution morphologique régionale correspondrait à une vitesse de colmatage supérieure à la vitesse de creusement (système en voie de fermeture, Mangin, 1983).

La charge acquise semble plus importante pendant les épisodes de crue qu'en périodes optimales d'eutrophisation pour lesquelles la forte sursaturation des eaux de Loire vis à vis de la calcite (valeurs négatives, Table 3) s'oppose en partie à la dissolution pendant le transit souterrain. Dans les deux cas, la dissolution, et donc la pérennité de la circulation karstique, semble pouvoir être imputée aux processus de minéralisation de la MO fluviatile, ce qui pose la question du rôle éventuel de l'anthropisation. La situation rencontrée pendant la période étudiée s'inscrit dans le cadre de la Loire moyenne valable depuis une dizaine d'années au moins (Lair and Sargos, 1993). Bien que le recul puisse paraître insuffisant, le processus nous semble être essentiellement d'origine naturelle, la charge organique mesurée en Loire étant conforme à celle de cours d'eau faiblement anthropisés (Meybeck, 1982).

Le rôle attribué aux processus de minéralisation de la MO fluviatile dans le système karstique du Val d'Orléans doit certainement opérer pour des systèmes analogues, citons par exemple celui de la Loue et du Doubs et celui de l'Aach et du haut Danube (Hoetzl, 1996; Chauve et al., 1987; Vançon, 1965). Ces systèmes possèdent cependant des bassins versants importants et sont alimentés seulement partiellement par les pertes des cours d'eau. D'autre part, ils sont situés dans la partie supérieure des cours d'eau avec une charge organique certainement inférieure à celle de la Loire moyenne. Le système du Val d'Orléans, comme réacteur traversé en permanence par un flux hydrique rapide dont on peut connaître le temps de transit ainsi 
que la composition à l'entrée et à la sortie, parait particulièrement propice, surtout en période estivale, à l'étude des transferts d'éléments à travers un interface redox.

\section{Conclusion}

Le système hydrologique du val d'Orléans, en période estivale, est un cas type d'alimentation karstique par des pertes de cours d'eau. Les eaux perdues en Loire en amont d'Orléans résurgent, notamment à la source du Loiret dite du "Bouillon", pratiquement sans aucun mélange. Cette caractéristique est illustrée de manière évidente par la correspondance entre les profils de chlorure enregistrés lors de périodes de crue; le déphasage des masses d'eau entre l'entrée et la sortie du système peut ainsi être estimé à environ 3 jours.

L'absence de mélange des eaux de Loire introduites dans le système avec par exemple des eaux infiltrées à travers la zone insaturée implique que la différence de chimisme des eaux à la résurgence ne résulte que de réactions dans le système.

Le modèle envisagé explique les principales caractéristiques acquises par les eaux résurgentes (anoxie, faible charge organique particulaire, alcalinité et teneur en calcium supérieures à celles des eaux de Loire) par un ensemble de réactions faisant intervenir l'oxydation de la matière organique fluviatile et la dissolution concomitante des calcaires.

La charge organique présente dans les eaux de Loire est suffisante pour alimenter le phénomène, la fraction particulaire paraissant être plus impliquée que la fraction dissoute.

Les mécanismes hétérotrophes mis en jeu sont l'aérobiose et la dénitrification sans que l'on ait pu formellement calculer leur part respective.

Les eaux de Loire infiltrantes ont une capacité de dissolution des calcaires très faible, voire négative, et la quasi totalité de la dissolution est une conséquence directe de l'oxydation de la matière organique fluviatile.

Cet ensemble d'observations fait considérer l'aquifère karstique du Val d'Orléans comme un réacteur particulièrement intéressant pour l'étude des transferts (éléments traces, composés organiques) à travers un interface redox.

\section{Remerciements}

Le conseil de M. Gil Michard (Université Paris VII-Denis Diderot) nous a été précieux pour la présentation de ce travail. Les résultats des mesures de $\mathrm{pH}$ et $\mathrm{O}_{2}$ dissous effectuées en continu en Loire à la station d'alerte implantée à Saint Denis de l'Hôtel, nous ont été obligeamment communiqués, en complément de nos propres données, par les services de $\mathrm{M}$. Maurice Rey (Lyonnaise des Eaux-Dumez).

\section{Références}

Bakalowicz, 1979

Bakalowicz, M. (1979) Contribution de la géochimie des eaux à la connaissance de l'aquifère karstique et de la karstification. Thèse, Université P\&M Curie, Paris 
Barillier, 1992

Barillier, A. (1992) Caractérisation et dynamique de la matière organique d'un milieu

fluvial anthropisé, la Seine. Thèse, Univerité P\&M Curie, Paris

Boitel, 1965

Boitel, R.-J. (1965) Le domaine de la Source. In La source du Loiret, pp. 23-27. Société

Orléanaise d'Histoire et d'Archéologie, Orléans

Bourg and Bertin, 1993

A.C.M. Bourg, C. Bertin

Quantitative appraisal of biogeochemical processes during the infiltration of river water into an alluvial aquifer

Environ. Sci. Technol., 27 (1993), pp. 661-666

Chauve et al., 1987

P. Chauve, P. Jacquemin, J. Mania

Un exemple d'alimentation induite d'un reservoir karstique par pertes de rivière en pays calcaire

Bull. Soc. Géol. Fr., 8 (1987), pp. 123-129

Chéry, 1983

Chéry, J.-L. (1983) Etude hydrochimique d'un aquifère karstique alimenté par perte de cours d'eau (la Loire): Le système des calcaires de Beauce sous le val d'Orléans. Thèse, Université d'Orléans

Chéry et al., 1982

Chéry, J.-L., Lelong, F. and Lepiller, M. (1982) Impact des apports de la Loire sur la qualité des eaux du système karstique des calcaires de Beauce sous le val d'Orléans. I. Aspects hydrodynamiques et hydrochimiques. In Actes du 3éme Colloque d'Hydrologie en Pays Calcaire, Annales Scientifiques, Géologie, Vol. 1, pp. 47-63. Univeristé de Besançon

Chesterikoff et al., 1992

A. Chesterikoff, B. Garban, G. Billen, M. Poulin

Inorganic nitrogen dynamics in the River Seine downstream from Paris (France)

Biogeochem., 17 (1992), pp. 147-164

Czysz et al., 1989

Czysz, W. et al. (1989) Waste Water Technology, ed. W. Fresenius, W. Schneider, B.

Böhnke and K. Pöppinghaus. Springer-Verlag, Berlin

Duchène, 1990

Duchène, P. (1990) Elimination de l'Azote dans les Stations d'Epuration Biologique des

Petites Collectivités, doc. tech. FNDAE No. 10. CEMAGREF-DICOVA, Antony, France 
Dupain, 1992

Dupain, S. (1992) Dénitrification biologique hétérotrophe appliquée au traitement des eaux d'alimentation: Conditions de fonctionnement et mise au point d'un procédé.

Thèse, Université Claude Bernard, Lyon

Espitalié et al., 1985

J. Espitalié, G. Deroo, F. Marquis

La pyrolyse Rock-Eval et ses applications

Rev. Inst. Fr. Pét., 40 (1985), pp. 563-579

Gonzalez, 1992

Gonzalez, R. (1992) Etude de l'organisation et évaluation des échanges entre la Loire moyenne et l'aquifère des calcaires de Beauce. Thèse, Université d'Orléans

Gounot, 1991

Gounot, A.-M. (1991) Ecologie microbienne des eaux et des sédiments souterrains.

Hydrogéologie, 239-248

Hoetzl, 1996

H. Hoetzl

Origin of the Danube-Aach system

Environ. Geol., 27 (1996), pp. 87-96

Ittekkot, 1988

V. Ittekkot

Global trends in the nature of organic matter in river suspensions

Nature, 332 (1988), pp. 436-438

Jacobs et al., 1988

L.A. Jacobs, H.R. von Gunten, R. Keil, M. Kuslys

Geochemical changes along a river-groundwater infiltration flow path: Glattfelden Switzerland

Geochim. Cosmochim. Acta, 52 (1988), pp. 2693-2706

Lair and Sargos, 1993

N. Lair, D. Sargos

A 10 year study at four sites of the middle course of the River Loire. I - Patterns of change in hydrological, physical and chemical variables in relation to algal biomass

Hydroécol. Appl., 5 (1993), pp. 1-27

Lepiller, 1980

Lepiller, M. (1980) Contribution de l'hydrochimie à la connaissance du comportement hydrogéologique des massifs calcaires. Etude de quelques systèmes karstiques du massif du Semnoz et de la région d'Annecy. Thèse, Univesité de Grenoble 
Livrozet, 1984

Livrozet, E. (1984) Influence des apports de la Loire sur la qualité bactériologique et chimique de l'aquifère karstique du val d'Orléans. Thèse, Université d'Orléans

Mangin, 1983

Mangin, A. (1983) L'approche systémique du karst, conséquences conceptuelles et méthodologiques. In Karst-Larra, Vol. 82, pp. 141-157. Diputacion Foral Navarra, Pampelune, Espagne

Mariotti, 1986

A. Mariotti

La dénitrification dans les eaux souterraines, principes et méthodes de son identification: une revue

J. Hydrology, 88 (1986), pp. 1-23

Mariotti et al., 1988

A. Mariotti, A. Landreau, B. Simon

${ }^{15} \mathrm{~N}$ isotope biogeochemistry and natural denitrification process in groundwater:

Application to the chalk aquifer of northern France

Geochim. Cosmochim. Acta, 52 (1988), pp. 1869-1878

Meybeck, 1982

M. Meybeck

Carbon, nitrogen, and phosphorus transport by major world rivers

Am. J. Sci., 282 (1982), pp. 401-450

Michard, 1989

Michard, G. (1989) Equilibres Chimiques dans les Eaux Naturelles. Publisud, Paris

Morel and Hering, 1993

Morel, M. M. and Hering, J. G. (1993) Principles and Applications of Aquatic Chemistry. Wiley, New York

Mulder et al., 1995

A. Mulder, A.A. van de Graaf, L.A. Robertson, J.G. Kuenen

Anaerobic ammonium oxidation discovered in a denitrifying fluidized bed reactor FEMS Microbiol. Ecol., 16 (1995), pp. 177-184

Plummer et al., 1976

Plummer, L. N., Jones, B. F. and Truesdell, A. H. (1976) WATEQF - a FORTRAN IV version of WATEQ, a computer program for calculating chemical equilibrium of natural waters, U. S. Geological Survey Water-Resources Investigations 76-13. NTIS, Springfield, USA 
R.P. Schwarzenbach, W. Giger, E. Hoehn, J.K. Schneider

Behavior of organic compounds during infiltration of river water to groundwater field studies

Environ. Sci. Technnol., 17 (1983), pp. 472-479

Scribe et al., 1995

Scribe, P., Pèpe, C., Albéric, P., Barreau, C., Fillaux, J., Saliot, A. and Bricket, J.-P. (1995) Biogéochimie de la matière organique des suspensions du Congo et de ses affluents: approche par les marqueurs moléculaires. In Grands bassins fluviaux périatlantiques: Congo, Niger, Amazone, ed J.-C. Olivry and J. Boulègue, pp. 83-95. ORSTOM, Paris

Sigg et al., 1992

Sigg, L., Stum, W. and Behra, P. (1992) Chimie des Milieux Aquatiques. Masson, Paris

Stum and Morgan, 1981

Stum, W. and Morgan, J. J. (1981) Aquatic Chemistry. Wiley, New York

Vançon, 1965

J.P. Vançon

Etude quantitative des pertes du Doubs et du Danube

Bull. Bur. Rech. Géol. Min., 4 (1965), pp. 151-167

Von Gunten et al., 1991

H.R. Von Gunten, G. Karametaxas, U. Krähenbühl, M. Kuslys, R. Giovanoli, E. Hoehn, R. Keil

Seasonal biogeochemical cycles in riverborne groundwater

Geochim. Cosmochim. Acta, 55 (1991), pp. 3597-3609

Zunino et al., 1980

C. Zunino, M. Bonnet, F. Lelong

Le Val d'Orléans: un exemple d'aquifère à alimentation latérale

C. R. somm. Soc. Géol. Fr., 5 (1980), pp. 195-199 